of the importance of MECC; this rose to 75\% (43/57) postsession. $84 \%$ (47/57) were confident with MECC skills presession, however this fell to $77 \%$ (44/57) post-session. Individuals reported questioning their ability to discuss healthy lifestyle behaviours and recognised the benefit of a structured approach to motivational interviewing. Those confident in their knowledge base of healthy lifestyles advice rose from $11 \%(6 / 57)$ to $29 \%(16 / 57)$. Confidence in the approach to conversation rose from $38 \%(21 / 57)$ to $56 \%$ (31/57); and in having a response to disengagement from patients and carers rose from $22 \%(12 / 57)$ to $52 \%$ (29/57). Knowledge of local resources, and where to signpost individuals to, also saw a rise from $16 \%(9 / 57)$ to $46 \%(26 / 57)$, and $8 \%$ (4) to $39 \%$ (22/57) respectively.

Conclusion Despite the evidence base for this Public health educational programme originating from adult research, adapted to aid professionals caring for children and young people has proved its worth as a useful resource. A collaborative approach with local public health teams would allow for this programme to be developed bespoke to local areas and would be a useful tool for motivational interviewing with children, young people and their carers to improve health lifestyles.

\section{G436(P) PREGNANT WOMEN'S VIEWS AND EXPERIENCES OF PERTUSSIS VACCINATION DURING PREGNANCY: A STUDY IN TAIWAN}

CY Lo, H Bedford, P Winyard, V Palit. Great Ormond Street Institute of Child Health, University College London, London, UK

\subsection{6/archdischild-2018-rcpch.425}

Introduction Pertussis resurgence has been a significant public health issue over the past decade in many countries including Taiwan. Maternal pertussis vaccination has been shown to be a safe and effective way to protect young infants who are not eligible to receive the vaccine. This is the new preventative vaccine policy in Taiwan and little was known about the pregnant women's attitude towards the pertussis vaccination.

Objective This study aims to evaluate pregnant women's attitudes about the maternal pertussis vaccine in Taiwan.

Method A cross-sectional questionnaire survey was conducted among the women accessing antenatal care at the Mackay Memorial Hospital in Taiwan. The vaccine uptake rate as well as how many women intended to be vaccinated were calculated and presented as percentages. Factors including demographic variables, awareness, accessibility and cost were analysed to determine the association with the women's intentions. The frequencies and percentages of the answers regarding preferred information sources and the reasons influencing their intentions were calculated.

Results 53 women were recruited.73.3\% of women intended to receive the vaccination but the actual uptake rate was $33.3 \%$. $64.7 \%$ of the participants were being informed of the vaccine by their obstetricians but only $13 \%$ had a meaningful discussion with their physicians. Demographic characteristics had no significant associations with their intention to be vaccinated. The most reliable information source was their obstetricians. Vaccine safety and efficacy concerns of vaccine, perception of disease severity and susceptibilities were the most important self-reported reasons influencing their intention.
Conclusion Vaccine uptake among the cohort was poor although they showed the relatively high intention to vaccinate. Healthcare professionals especially obstetricians in Taiwan need to recommend the maternal pertussis vaccine and provide accurate information to increase the success of this important vaccination programme.

\section{G437(P) AWARENESS AND UPTAKE OF THE DEPARTMENT OF HEALTH RECOMMENDATIONS ON VITAMIN D SUPPLEMENTATION IN CHILDREN UNDER 5}

RM O'Dea, R Hulbert, K Fraser. Children and Young People's Health Services, Cambridgeshire Community Services NHS Trust, Huntingdon, UK

\subsection{6/archdischild-2018-rcpch.426}

Background The Department of Health currently recommends dietary vitamin $\mathrm{D}$ supplementation for all children under the age of 5 years.

Aims

- Assess parents' awareness of the recommendations for vitamin D supplementation.

- Assess current uptake of vitamin D supplementation in children under 5 .

- Investigate barriers to vitamin D supplementation.

Methods This cross-sectional observational study used paper questionnaires to collect data over a three week period. Questionnaires were distributed to all parents and carers attending the paediatric outpatients of a district general hospital with a child under the age of 5. An information sheet outlining the current vitamin D supplementation recommendations was provided.

Results 28 responses were received; 54\% of parents were aware of the Department of Health recommendations for vitamin D supplementation, and $46 \%$ were not.

$54 \%$ of respondents were not meeting recommendations for vitamin D supplementation.

Of those not meeting the recommendations for vitamin D supplementation, $73 \%$ of parents were unaware of the current recommendations. Having now been made aware of the recommendations, these parents unanimously reported that they would consider giving vitamin D supplementation in the future.

Parents who were aware of the recommendations but not giving vitamin D supplementation reported reasons including: dislike of the vitamin drops by the child, low prioritisation to acquire the drops and the belief that varied diet, breastfeeding and outdoor play is sufficient. $75 \%$ of these parents would now consider giving vitamin $\mathrm{D}$ supplementation following completion of the survey.

Conclusions A lack of awareness of the current Department of Health recommendations has been demonstrated in our parent population. Parents who were previously unaware of the recommendations would now consider giving vitamin D supplements to their child; this suggests that parents and carers of young children are receptive to Public Health recommendations and emphasises the need for effective communication of recommendations to the target audience.

Ongoing work involves data collection in a larger cohort of patients to confirm these findings. The knowledge of healthcare professionals in relation to these guidelines will also be explored. 